\title{
Les politiques publiques agricoles en France. Une évaluation au milieu du gué?
}

Marielle Berriet-Solliec, Marc Guérin et Dominique vollet

\section{Q OpenEdition}

1 Journals

Édition électronique

URL : http://journals.openedition.org/economierurale/3208

DOI : $10.4000 /$ economierurale.3208

ISSN : 2105-2581

Éditeur

Société Française d'Économie Rurale (SFER)

Édition imprimée

Date de publication : 5 juillet 2005

Pagination : 89-95

ISSN : 0013-0559

Référence électronique

Marielle Berriet-Solliec, Marc Guérin et Dominique vollet, « Les politiques publiques agricoles en

France. Une évaluation au milieu du gué ? ", Économie rurale [En ligne], 286-287 | Mars-juin 2005, mis en ligne le 05 mars 2005, consulté le 24 septembre 2020. URL : http://journals.openedition.org/ economierurale/3208; DOI : https://doi.org/10.4000/economierurale.3208

Ce document a été généré automatiquement le 24 septembre 2020.

(c) Tous droits réservés 


\title{
Les politiques publiques agricoles en France. Une évaluation au milieu du gué ?
}

\author{
Marielle Berriet-Solliec, Marc Guérin et Dominique vollet
}

Historiquement en France, à partir de 1990, l'évaluation a été associée à la réforme de l'État, dont elle a constitué une des composantes importantes. Parallèlement à ce volontarisme, l'évaluation résulte aussi d'une impulsion européenne affirmée lors de la réforme des fonds structurels de 1988. Les politiques agricoles ont été particulièrement concernées par cette évolution, dans un souci d'amélioration de leur condition de mise en œuvre comme de l'optimisation de leurs effets. Il nous a, dès lors, semblé important d'amorcer une analyse critique sur ces années passées en y jetant le regard croisé du scientifique impliqué dans des recherches sur les démarches et méthodes d'évaluation et de l'expert engagé dans des procédures évaluatives ${ }^{1}$. Nous nous sommes assignés deux principaux objectifs pour cette analyse: (i) après quinze ans de pratiques de l'évaluation, dresser un premier bilan de ces opérations en soulevant un triple point de vue : institutionnel, méthodologique et opérationnel (diffusion et portée des résultats des évaluations); (ii) au terme d'une période pionnière, s'interroger sur le passage à une phase de maturité de ces pratiques et mettre en perspective les évolutions observées. Assiste-t-on à une inflexion de l'évaluation par rapport aux pratiques antérieures? Un fossé entre les analyses scientifiques et les démarches pragmatiques ne commencerait-il pas à se creuser?

\section{Quinze ans après... l'évaluation à l'heure du bilan}

\section{Le rôle moteur de l'Union européenne}

Depuis le lancement de l'évaluation sous l'égide du Conseil scientifique de l'évaluation (CSE) en 1990 puis celle du Conseil national de l'évaluation (CNE) et du Commissariat général du plan, l'Europe joue un rôle moteur de par l'obligation d'évaluation de la 
plupart des programmes co-financés (le Parlement français ne jouant qu'un rôle restreint). À l'échelle des régions, les préfectures, à travers les SGAR (Secrétariat général aux affaires régionales) et les services déconcentrés en région, s'impliquent dans le suivi des évaluations en liaison avec les Conseils régionaux. L'évaluation a été impulsée par la Commission, notamment par la diffusion des documents MEANS (Méthodes d'évaluation des actions de nature structurelle) ${ }^{2}$. Actuellement, elle continue largement à diffuser largement la culture de l'évaluation ${ }^{3}$.

Deux conceptions de l'évaluation co-existent. D'une part, celle interne à la Commission conçoit l'évaluation comme le travail d'un bureau d'étude. Le suivi est assuré, en principe, par un groupe technique composé uniquement d'administratifs. D'autre part, dans la tradition de l'État républicain, une culture française de l'évaluation bâtie comme un lieu de dialogue interministériel, insiste sur le rôle d'une instance d'évaluation pluraliste. Les membres de l'instance sont choisis intuitu personae, en raison de leur expérience et de leur indépendance d'esprit.

4 Alors que la conception portée par l'Union européenne accorde aux cabinets d'étude un rôle crucial dans l'expertise, l'approche française privilégie davantage un équilibre subtil entre la mise au point d'un diagnostic partagé, appropriation des résultats de l'étude et l'indépendance des conclusions.

5 Cette spécificité nationale est-elle actuellement remise en cause? Au contraire, n'existe-t-il pas encore en France, pour les ministères techniques, une possibilité de « compléter » les approches communautaires ? L'expérience récente de l'évaluation du Règlement de développement rural (RDR) (2001-2003), notamment en ce qui concerne le "lot de synthèse ", montre qu'il est possible, tout en respectant la démarche communautaire de conférer à un comité de pilotage un rôle assez important qui dépasse celui des comités techniques habituels (notamment dans la définition du cahier des charges).

\section{Les enseignements en termes méthodologiques}

6 Au regard de l'expérience française, les démarches d'évaluation ont, semble-t-il, fortement progressé. Pour une majorité d'acteurs, l'évaluation n'est plus perçue comme la simple collecte d'indicateurs. L'analyse des évaluations agricoles et rurales conduites depuis les années 1990 indique que pour un même questionnement relatif à la cohérence, la pertinence et l'efficacité des politiques, il existe une grande hétérogénéité de la précision des réponses par les différents prestataires.

7 Comme le montrent les dernières évaluations du RDR et du CTE (Contrat territorial d'exploitation), la phase initiale d'analyse de politique visant à expliciter la théorie d'action (c'est-à-dire les relations de causes à effets implicitement ou explicitement contenues dans la politique) paraît de plus en plus systématiquement réalisée. Ce travail permet de définir différents objectifs qui sont ensuite hiérarchisés sous formes d'arbre d'objectifs'.

Bien que ce type de formalisation présente des intérêts certains (représentation synthétique de l'action publique, analyse de la cohérence de la politique, sélection des indicateurs pertinents), dans certaines évaluations récentes, sa réalisation se réduit trop souvent à la reprise approximative des principaux alinéas des dispositions législatives et/ou réglementaires à l'origine de la politique. Pourtant, elle éviterait de 
tomber dans une chausse-trappe classique en évaluation: la collecte de nombreux indicateurs souvent mal renseignés et peu structurés.

En ce qui concerne les méthodes quantitatives, l'impact d'une politique peut être approché de manière dite "naïve » en comparant les lieux ou les individus concernés par la politique et les autres. Cette démarche est généralement biaisée (par un «biais de sélection») car l'un des scénarios (le contrefactuel) n'est pas observé. Deux méthodes existent: utiliser des expériences expérimentales ou construire des expériences contrôlées. En France, pour ce second type de méthode, des progrès significatifs sont à noter comme l'utilisation de méthodes statistiques de correction des biais de sélection. Toutefois, bien que présentant une littérature économique abondante et des potentialités fortes ${ }^{5}$, les outils de la micro-économie et la macroéconomie restent très peu mobilisés dans les évaluations appliquées à la politique agricole et de développement rural, à l'exception de quelques travaux pionniers ${ }^{6}$. En macro-économie, l'utilisation du modèle input-output ou de matrices de comptabilité sociale reste marginale (voir à ce sujet l'évaluation du Plan de développement des zones rurales en Bretagne), ce qui n'est d'ailleurs pas le cas des autres pays européens (notamment anglo-saxons ou italiens) ${ }^{7}$. L'évaluation des politiques publiques reposant sur des bases micro-économiques solides est également très peu présente (même en utilisant des fonctions de production et de demande en information parfaite). Par exemple, malgré les limites de la méthode, l'estimation des préférences des consommateurs sur des marchés hypothétiques (exemple : évaluation contingente) ou à partir de préférences révélées (méthode des prix hédonistes) relève essentiellement du domaine de la recherche et reste très peu utilisée pour les évaluations de politiques agricoles.

10 Fait récurrent, la mise en œuvre concrète de l'évaluation rencontre, en outre, comme obstacle majeur, la disponibilité de données socio-économiques, notamment à l'échelle infra-nationale. Ces difficultés ont pour origine une lacune persistante dans le système d'information statistique français concernant la territorialisation des données ${ }^{8}$. Elles constituent un obstacle majeur à l'amélioration de la qualité des évaluations et un facteur limitant pour la mise en œuvre de méthodes plus sophistiquées. Pour l'évaluateur, cette collecte d'informations, souvent de première main, nécessite un temps non négligeable qui empiète souvent sur la phase d'évaluation proprement dite.

\section{Les enseignements opérationnels}

11 Pour l'instant, la mise en pratique de l'évaluation en France s'est accompagnée davantage de changements dans les pratiques administratives que dans les fondements et les modalités des politiques elles-mêmes.

12 Actuellement, au ministère de l'Agriculture, de l'Alimentation, de la Pêche, et de la Ruralité (MAAPR), l'évaluation est spécifiquement prise en charge par un bureau chargé de l'évaluation. Trois grands types d'évaluations sont mis en œuvre au MAAPR :

- des évaluations interministérielles comme par exemple les politiques de développement rural en 2003, la politique de la montagne en 1999), (le bureau chargé de l'évaluation et les directions techniques étant associées, par exemple pour fournir un rapporteur), 
- des évaluations ministérielles (au nombre d'une quinzaine environ depuis 2000) demandées par les directions centrales ou les services déconcentrés (DRAF) et pilotées par les directions à l'origine de la demande,

- des évaluations européennes (comme l'évaluation à mi-parcours du RDR) dont la mise en œuvre est assurée par le bureau chargé de l'évaluation.

L'évaluation des programmes européens structure fortement l'activité d'évaluation du MAAPR (mise en place de dix comités nationaux d'évaluation pour l'évaluation du RDR). Inspirée par les pratiques des évaluations interministérielles coordonnées par le Plan jusqu'en 2003, l'ouverture des instances nationales et régionales d'évaluation à des acteurs non agricoles (ministère en charge de l'Environnement, associations, etc.) représente une innovation certaine.

Le faible impact des évaluations sur les politiques elles-mêmes est sans doute à rechercher dans le caractère encore trop confidentiel des rapports d'évaluation et la faible portée des résultats. La mise en ligne sous Internet des rapports d'évaluation du RDR représente une évolution importante dans les pratiques. L'évaluation peut, en effet, être considérée comme un outil de dialogue avec la société civile et d'ouverture de certaines administrations, à condition d'assurer une diffusion large des résultats obtenus.

Quinze ans après le lancement d'une démarche d'évaluation coordonnée, le dispositif français d'évaluation se réorganise progressivement. Il n'existe plus actuellement d'organisme interministériel en charge de l'évaluation qui, outre un certain volontarisme, avait pour avantage de mettre l'accent dans l'analyse comme dans les préconisations, sur la nécessaire coordination interministérielle dans la conception et la mise en œuvre des dispositifs publics.

En revanche, en 2003, le ministère de la Fonction publique s'est vu confirmer sa mission de favoriser le développement de l'évaluation des politiques publiques. Parallèlement le rôle du Parlement, naturellement orienté vers le contrôle, est en progression même s'il reste encore discret. On peut, dès lors, se demander quelles seront les évolutions dans les services déconcentrés et les répercussions sur les pratiques actuelles, en sachant que se structurent toujours davantage les services d'évaluation au sein des collectivités territoriales.

17 Malgré la diffusion de la culture de l'évaluation tant au niveau régional (via les SGAR) qu'au niveau national dans les différents ministères, une autre interrogation demeure. Elle porte sur le risque que l'évaluation, sans relais institutionnel structuré, devienne encore plus une affaire de spécialistes avec une délégation des missions d'évaluation confiée aux cabinets privés selon une logique d'expertise standardisée, avec en corollaire un fossé croissant avec la recherche.

\section{Vers un fossé croissant entre théorie et pratique de l'évaluation?}

\section{Quelle place pour la recherche finalisée dans le marché de l'évaluation?}

Pour l'observateur français qui analyse la réponse aux appels d'offre concernant les domaines agricoles et de développement rural, le bilan montre que les chercheurs sont 
de moins en moins nombreux à participer à des opérations concrètes d'évaluation. Les établissements de recherche ou les établissements d'enseignement supérieur agronomique sont ainsi rarement associés et sont exceptionnellement pilotes, la quasitotalité des évaluations étant réalisée par des cabinets d'étude. Par exemple, sur les 21 évaluations régionales CTE-MAE, une seule a été réalisée par des enseignantschercheurs, en région Rhône-Alpes. Deux principales raisons peuvent être avancées : d'une part, les délais de réalisation relativement courts (six mois à un an maximum), par conséquent difficilement compatibles avec les calendriers de travail des chercheurs, d'autre part, la difficulté de valoriser ces travaux selon les normes de publication scientifique.

Cette réalisation des évaluations par des cabinets d'étude se traduit par une grande diversité des travaux. Dans le cas des CTE-MAE, une synthèse qualitative des rapports rendus par les cabinets d'étude souligne leur très grande hétérogénéité. Tandis que certains relèvent de démarches scientifiques rigoureuses, d'autres souffrent de grandes faiblesses méthodologiques malgré les efforts de la Société française de l'évaluation (SFE) pour mettre en avant des "standards de qualité». Absence d'analyse de la politique, faible recul par rapport aux dires des acteurs, manque de liens entre les éléments quantitatifs et qualitatifs rendant l'administration de la preuve délicate ${ }^{9}$ sont les principales critiques relatives à ces travaux.

La saturation des capacités de réponse des cabinets privés n'explique que pour partie ce bilan mitigé. En matière de prestation de service à court terme et sans effort d'organisation spécifique des conditions sociales d'émergence des relations d'échange de service, le libre et unique jeu des forces du marché présente des limites certaines. Le souhait d'obtenir de nouveaux marchés d'évaluation peut rendre les prestataires de service très soucieux de ne pas trop déplaire aux commanditaires.

Le maintien d'une expertise publique en matière d'évaluation au sein de l'État ou des établissements publics apparaît dès lors indispensable pour établir un « référentiel » en termes de démarches méthodologiques. En l'absence de ces normes, toute régulation devient difficile. Dans ce but, des relations plus étroites sur des bases claires et des formes nouvelles d'associations entre recherche publique et cabinets d'étude sont sans doute à expérimenter. Dans le cadre d'une véritable coopération, chacun des partenaires peut apporter son savoir-faire tant du point de vue conceptuel que méthodologique.

La compatibilité des deux approches reste toutefois souvent difficile. En effet, le monde de la recherche est soumis à des standards internationaux qui se traduisent par différentes contraintes de publications ou encore d'organisation en réseaux, peu compatibles avec les pratiques d'évaluation. En économie, par exemple, les développements récents en termes d'évaluation des politiques publiques, dans la lignée des travaux de micro-économie d'Heckmann, mobilisent aujourd'hui des techniques économétriques pointues et difficilement applicables directement par des évaluateurs (du moins par les cabinets spécialisés).

\section{Quelle utilité des travaux d'évaluation?}

En vue d'accroître encore davantage la traduction opérationnelle des évaluations, il semble indispensable de s'interroger sur leurs rôles, à la fois sur le court et le long terme. 
- Une aide à la décision : ce rôle de l'évaluation est particulièrement manifeste au niveau européen pour la Commission. Les évaluations ex post parvenant après l'élaboration du nouveau programme, les évaluations à mi-parcours se révèlent en fait celles ayant les plus grandes implications pour le programme suivant.

- Un catalyseur dans la diffusion des idées et dans les effets d'apprentissage : l'impact des évaluations est sans doute à rechercher à long terme. Les analyses, les recommandations modifient progressivement les discours ambiants, rendant difficiles certaines décisions. L'élaboration d'instances pluralistes associant des personnalités aux capacités d'expertise diversifiées fait inéluctablement évoluer les connaissances communes et les pratiques.

\section{Quelles perspectives?}

Sans remettre en cause ni les pratiques des cabinets d'étude, ni le caractère fondamental des recherches en évaluation des politiques publiques, la question des intermédiaires entre ces « deux mondes » mérite d'être soulevée.

En termes de conception même de l'évaluation, celle-ci ne peut se réduire ni à un simple contrôle ${ }^{10}$, ni à une étude stratégique, ni à un exercice de prospective. Il apparaît important de défendre les intérêts de l'analyse et de l'évaluation de l'intervention publique qui intègre tant les efforts de conceptualisation et de construction de référentiel pour porter un jugement de valeur que les aspects quantitatifs pour mesurer plus rigoureusement les effets de la politique étudiée. En termes organisationnels, il faut souligner la possibilité de faire jouer les synergies via des formules originales comme des structures d'étude de certains établissements ${ }^{11}$ ou encore des expériences de partenariats entre administratifs, recherche et enseignement supérieur. D'autres opérations partenariales sont à encourager qui peuvent notamment se traduire par l'association des cabinets privés à la définition des problématiques et la mise au point de méthodes « lourdes ».

Ceci nécessite une connaissance mutuelle préalable des institutions et un réel partage des acquis de la recherche menée de part et d'autre, des méthodes respectives mobilisées et des formes diverses de valorisation. Ces collaborations ouvrent des perspectives scientifiques intéressantes pour conceptualiser, par exemple, les théories d'action et les relations de cause à effet ou encore pour modéliser des outils économétriques récents.

\section{Conclusion}

27 Dans un contexte marqué par la multiplicité des acteurs et l'imbrication des procédures, l'évaluation apparaît plus que jamais fondamentale pour l'action publique. $\mathrm{Au}$ terme de ces premiers éléments de bilan sur l'évaluation en France des politiques agricoles en France, il nous semble important de souligner deux écueils qui apparaissent comme autant de pierres d'achoppement pour le développement de l'évaluation. Le premier est lié à l'absence de coordination interministérielle de l'évaluation, le second à l'importance accordée à la programmation plutôt qu'aux analyses rétrospectives.

Depuis une quinzaine d'années, le diptyque instance d'évaluation et chargé d'évaluation a constitué la principale originalité de «l'évaluation à la française ». Il 
constitue, selon nous, l'une des garanties de la qualité des travaux d'évaluation. Actuellement, en France, il n'existe pas d'organisme public en charge de l'évaluation interministérielle et le système du pilotage des évaluations par des instances pluralistes risque de tomber en désuétude. Cette absence laisse ainsi courir le risque de ne plus intégrer la pluralité des jugements et des expertises, de perdre la garantie d'une certaine objectivité et de se priver des avancées de la recherche en sciences sociales. Le relais est certes pris à l'échelle des collectivités territoriales mais ce développement des instances d'évaluation à l'échelle décentralisée a besoin d'être encouragé pour généraliser la démarche au-delà des quelques collectivités. Ces collectivités, fortement mobilisées, ont d'ailleurs besoin de références communes notamment en ce qui concerne les procédures d'évaluation des politiques nationales et, de manière plus générale, pour permettre la capitalisation des connaissances, faciliter les comparaisons entre territoires ainsi que l'échange des pratiques.

Dans un contexte d'incertitude liée intrinsèquement à l'action collective, l'efficacité de l'État passe non seulement par une maîtrise de ses dépenses mais aussi par la compréhension des conséquences de ses interventions et une capacité d'anticipation et d'adaptation à des situations nouvelles. C'est l'intelligibilité de l'action publique et ses impacts sur le développement socio-économique de notre pays qui est en jeu

\section{NOTES}

1. Les propos tenus dans cette tribune n'engagent que leurs auteurs.

2. . Commissioneuropéenne. Évaluer les programmes socio-économiques. Office des publications officielles des Communautés européennes, Collection MEANS, Luxembourg, 1999, 227 p.

3. . Voir notamment Evaluation of socio-economic Development - The Guide (http:// www.evalsed.info).

4. . Daucé P. L'évaluation des politiques communautaires de développement régional. Enjeux, méthodes, résultats. L'exemple de l'objectif $5 b$ en Bourgogne. Revue d'Economie Régionale et Urbaine, 1998, n³ 3. p. 379-394.

5. . Voir à ce sujet, Magnac T. L'apport de la micro-économétrie à l'évaluation des politiques publiques. INRA, Cahiers d'Economie et Sociologie Rurales, 2000, nº 54, p. 89-113.

6. . Berriet-Solliec M., Daucé P., Léon Y., Schmitt B. Vers un indicateur de croissance économique locale pour l'évaluation des politiques de développement rural.Revue d'Économie Régionale et Urbaine, 2001, n 3, p. 415-443. À noter que dans le cadre de la préparation du RDR 2007-2013, le ministère de l'Agriculture a intégré l'importance, en amont de la mise en œuvre des dispositifs, de la construction d'un référentiel et de la constitution d'une base de données appropriées pour la prise en compte de ces biais de sélection, et conçoit dès à présent un cadre méthodologique d'appui aux futures évaluations de ce règlement.

7. . En matière de politique rurale, les méthodes classiques un peu élaborées ne sont guère plus utilisées. Ainsi les projets, qui se prêteraient assez bien à une analyse coûts/avantages comme par exemple les aménagements touristiques, n'en sont jamais l'objet. 
8. . Le rapport Perrin Les politiques de développement rural souligne les carences du système d'information et préconise son amélioration. $C f$. Commissariat général du plan, Documentation française, 2003, $479 \mathrm{p}$.

9. . Nous avons même relevé, entre trois rapports régionaux réalisés par le même cabinet, des passages strictement identiques concernant la mise en œuvre départementale...

10. . À noter, la loi organique du $1^{\mathrm{er}}$ août 2001 relative aux lois de finances, semble orienter davantage l'évaluation vers la programmation ex ante que vers l'évaluation a posteriori. Elle met essentiellement l'accent sur les opérations de contrôle de gestion et de mesure de la performance 11. . Le CESAER de Dijon (Centre d'économie et de sociologie appliquées à l'agriculture et aux espaces ruraux) possède ainsi une cellule des études, qui se veut une structure de transferts de certains acquis de la recherche, en même temps qu'un partenaire pour participer concrètement à des évaluations, dans le respect des règles de la concurrence et des codes des marchés publics.

\section{AUTEURS}

\section{MARIELLE BERRIET-SOLLIEC}

ENESAD, Centre d'économie et de sociologie appliquées à l'agriculture et aux espaces ruraux (CESÆR), Dijon

MARC GUÉRIN

Cemagref, Antony

DOMINIQUE VOLLET

Cemagref, Clermont-Ferrand 